\section{International Scientific Journal Theoretical \& Applied Science}

\author{
p-ISSN: 2308-4944 (print) e-ISSN: 2409-0085 (online) \\ Year: $2015 \quad$ Issue: $01 \quad$ Volume: 21 \\ Published: $30.01 .2015 \quad$ http://www.T-Science.org
}

SECTION 4. Computer science, computer engineering and automation.
Manat Beyshenovich Tlebayev

Doctor of engineering science, Professor, Academician of Kazakh National Academy of Natural Sciences, Head of the department «Computer engineering and informatics» Taraz State University named after M.Kh. Dulati, Kazakhstan

Pavel Nikolayevitch Shevtsov postgraduate student,

Bachelor of computer science, Taraz State University named after M.Kh. Dulati, Kazakhstan pasha369@mail.ru

\title{
DEVELOPMENT OF MEANS OF TRAINING STUDENTS IN THE SUBJECT "COMPUTER MODELING"
}

Abstract: In this study, a model of student learning with the use of modern information and computer tools in education, in the discipline of Computer simulation.

Key words: training, modeling, model, deterministic model.

Language: Russian

Citation: Tlebayev MB, Shevtsov PN (2015) DEVELOPMENT OF MEANS OF TRAINING STUDENTS IN THE SUBJECT "COMPUTER MODELING". ISJ Theoretical \& Applied Science 01 (21): 198-203. doi: http://dx.doi.org/10.15863/TAS.2015.01.21.33

\section{РАЗРАБОТКА СРЕДСТВ ОБУЧЕНИЯ ПО ДИСЦИПЛИНЕ "КОМПЬЮТЕРНОЕ МОДЕЛИРОВАНИЕ"}

Аннотация: В данном исследовании предлагается модель обучения студентов с использованием современных информационных и компьютерных средств в образовании, по дисциплине Компьютерное моделирование.

Ключевые слова: обучение, моделирование, модель, детерминированнье модели.

В настоящее время моделирование составляет неотъемлимую часть современной науки, важность которой приближается к традиционным экспериментальным и теоретическим методам научного познания [1].

Цель курса - расширить представления студентов о моделировании как методе научного познания, и использование компьютера как инструмента научно-исследовательской деятельности.

Учебно-методический комплекс по курсу "Компьютерное моделирование" включает в себя университетскую программу расчитаную на 1 семестр обучения в высшем учебном заведении [2-7].

Программа состоит из пяти крупных разделов. Навигация по всей этой системе очень проста и представляет собой классическую схему представления в виде меню, расположенного сверху на каждой странице. Переходы между различными страницами осуществляются, в основном, с помощью этого меню. 


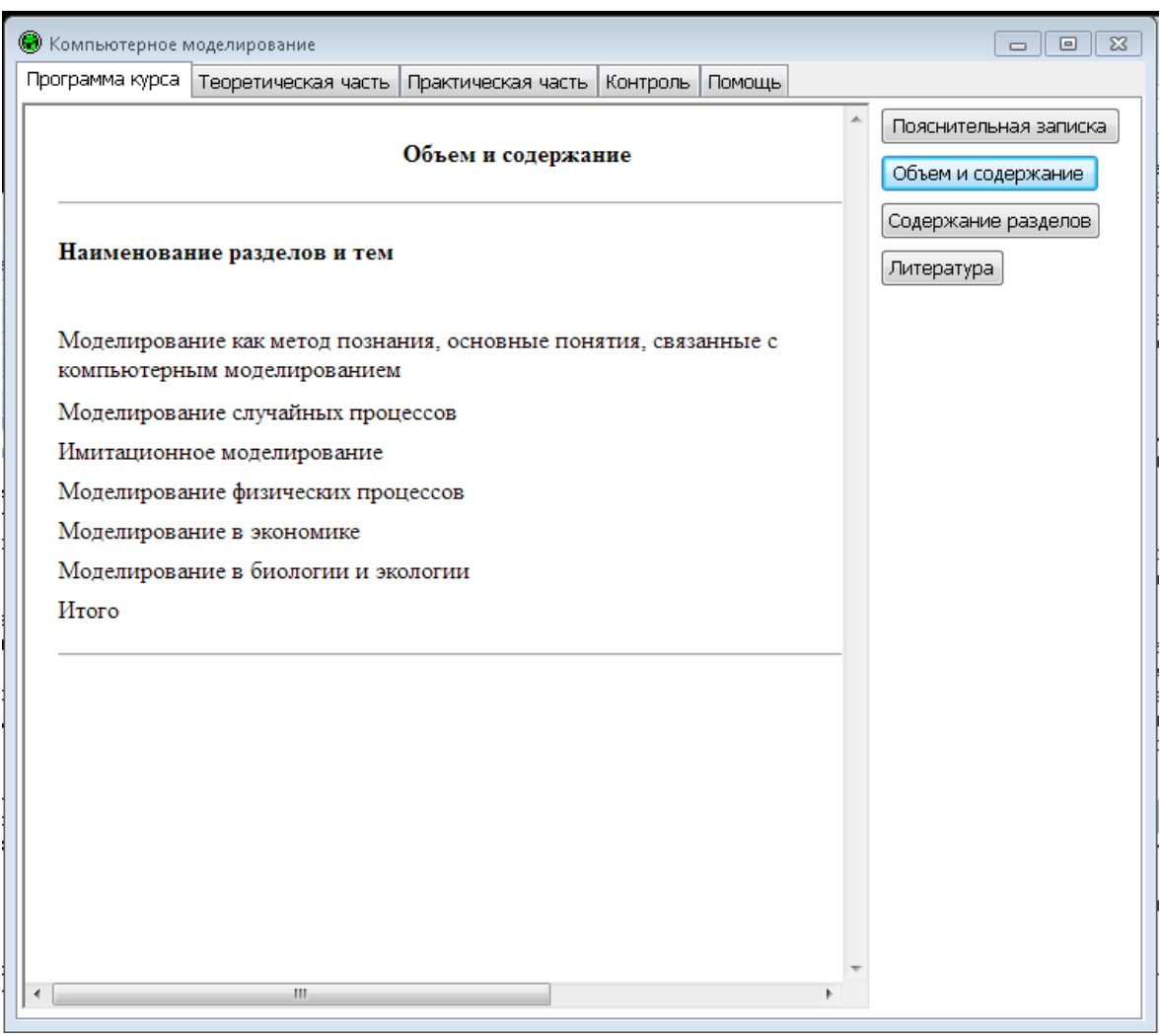

Рисунок 1 - Основное окно программы с навигацией по разделам.

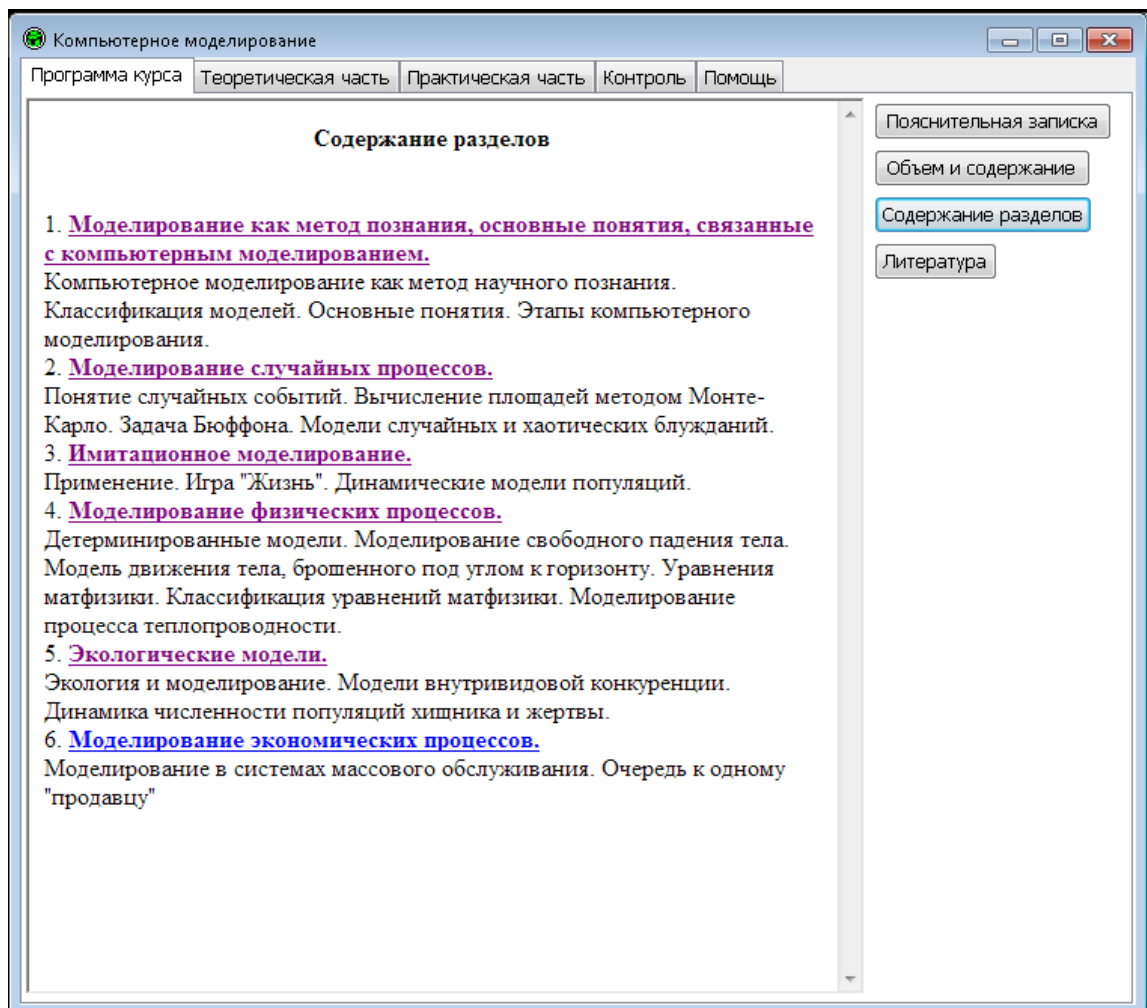

Рисунок 2 - Содержание разделов дисциплины. 
Меню позволяет перейти с любого места комплекса на любое другое, не используя при этом промежуточные страницы. Это представляет пользователю удобный и легко запоминаемый интерфейс программы и быстрые переходы между любыми страницами, это занимает не более 3-4 секунд.

Комплекс содержит не только учебные, но и множество вспомогательных материалов. К таковым относятся: глоссарий, электронный учебник, богатая библиотека выполненных компьютерных моделей, большой список рекомендуемой литературы.

К учебным же относятся уроки, лекции, лабораторные занятия, проектные задания, тестирования, экзаменационные вопросы.

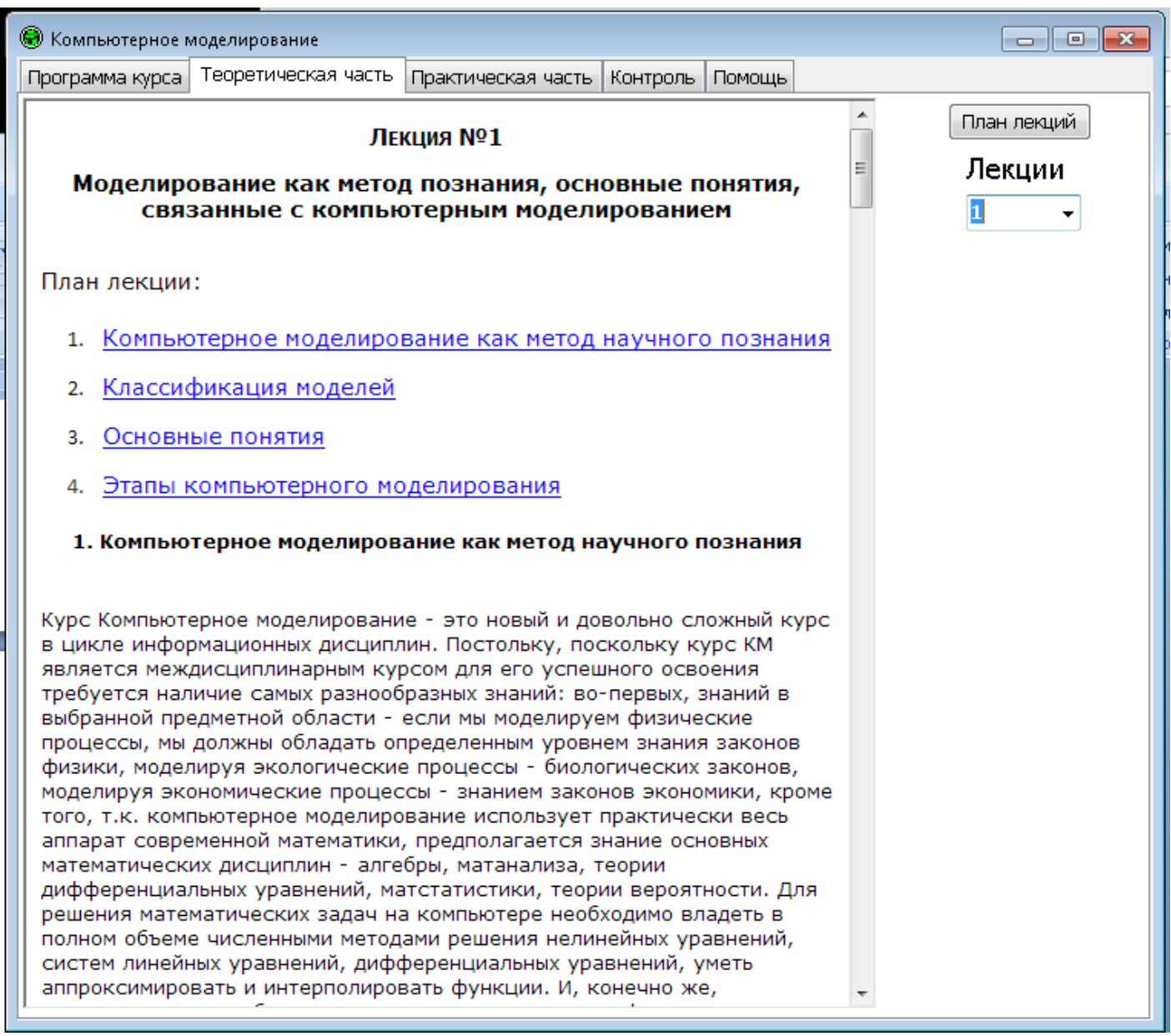

Рисунок 3 - Теоретическая часть, лекционные материалы. 


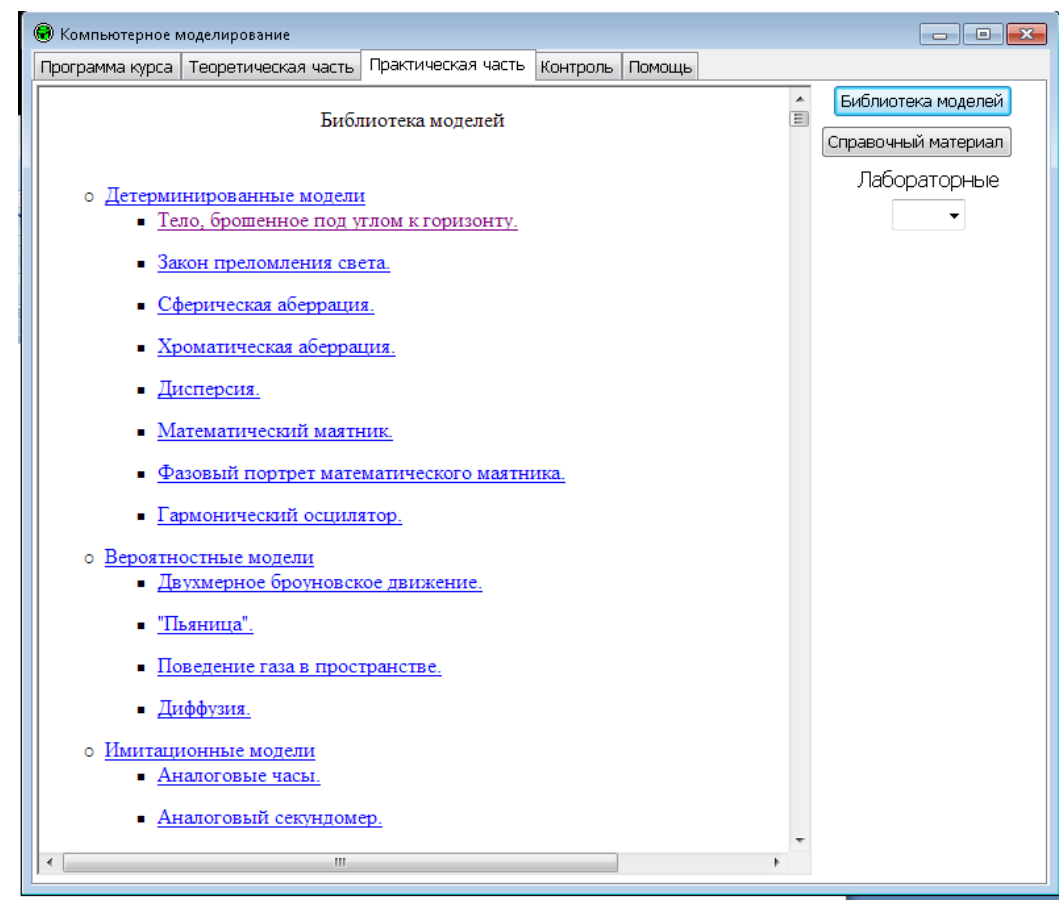

Рисунок 4 - Содержание практических заданий.

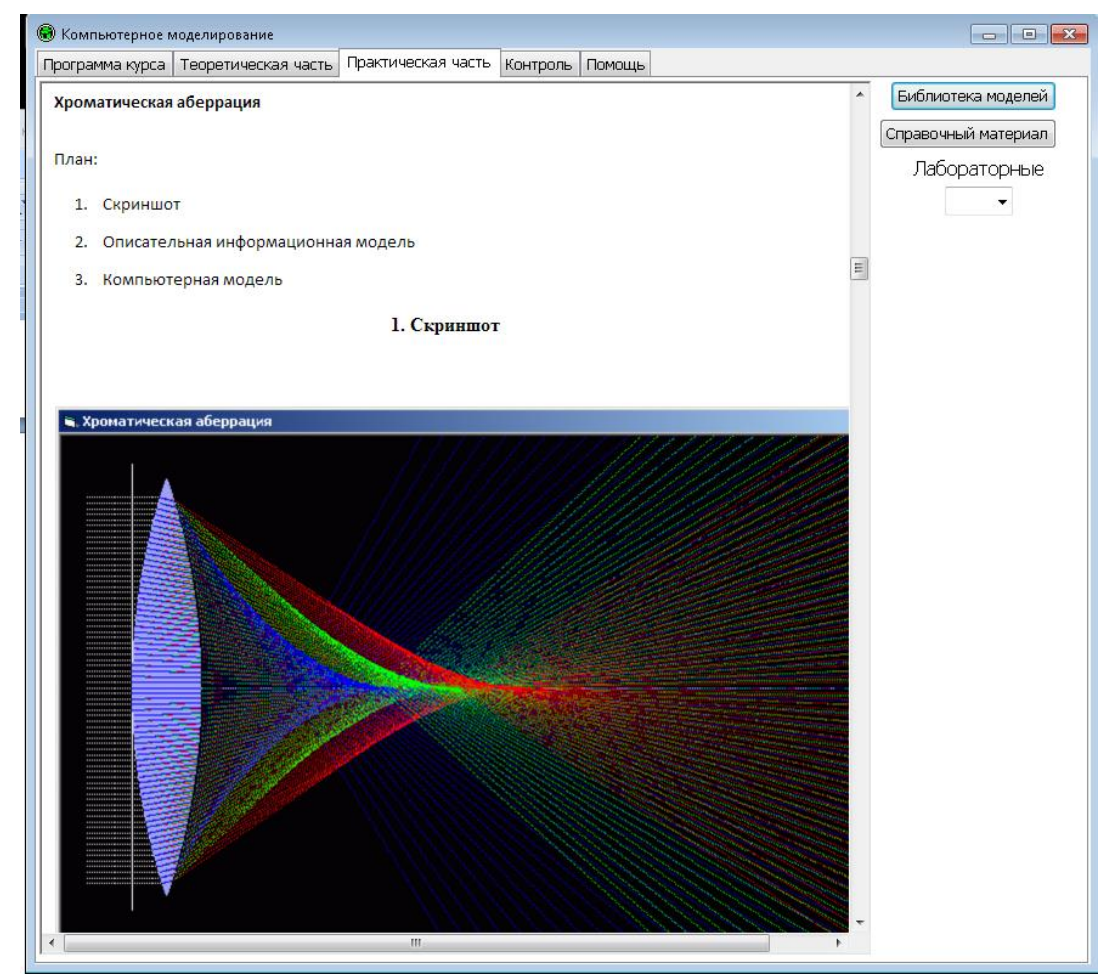

Рисунок 5 - Пример проведения лабораторной работы.

Основной метод контроля в учебнометодическом комплексе является метод проектов и лабораторных работ, так же не исключается непосредственное участие преподавателя для оценки результатов. 


\begin{tabular}{|c|c|c|}
\hline (9) Компьютерное моделирование & & 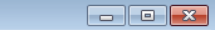 \\
\hline \begin{tabular}{l|l|l|l|} 
Программа курса & Теоретическая часть & Практическая часть & Контроль \\
\end{tabular} & & \\
\hline 3. Спроектировать пользовательский интерфейс программы моделирования, обрашая & - & Библиотека моделей \\
\hline 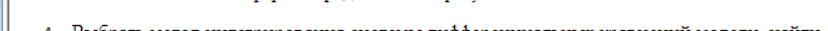 & & Справочный материал \\
\hline $\begin{array}{l}\text { 4. Выборать метод интегрирования системы дифференциальных уравнений модели, найти } \\
\text { или разработать программу интегрирования с заданной точностью. }\end{array}$ & & Лабораторные \\
\hline 5. Произвести отладку и тестирование полной программы. & & - \\
\hline 6. Выполнить конкретное задание из своего варианта работы. & & \\
\hline 7. Качественно проанализировать результаты моделирования. & & \\
\hline 8. Создать текстовый отчет по лабораторной работе, включающий: & & \\
\hline - постановку задачи и описание модели; & & \\
\hline - результаты тестирования программы; & & \\
\hline - результаты, полученные в ходе выполнения задания (в различных формах); & & \\
\hline - качественный анализ результатов. & & \\
\hline 5. Список лабораторных работ по темам & & \\
\hline - Дабораторная 1 - Моделирование движения тел в среде с үчетом трения & & \\
\hline - Лабораторная 2 - Моделирование движения небесных тел и заряженных частиц & & \\
\hline - Лабораторная3 - Моделирование колебательных процессов & & \\
\hline - Лабораторная4 - Моделирование физических процессов в приближении сплошной & & \\
\hline среды & 7 & \\
\hline - Лабораторная5 - Моделирование динамики численности популяций & & \\
\hline - Лабораторная6 - Моделирование очередей в системах массового обслуживания & $\equiv$ & \\
\hline - Лабораторная 7 - Моделирование различных случайных процессов & & \\
\hline & - & \\
\hline
\end{tabular}

\section{Рисунок 6 - Контроль по средством лабораторных работ.}

В каждой из программ имеется список проектов для самостоятельной работы. Эти проектные задания подразумевают либо построение новых информационных моделей, либо усовершенствование уже сделанных, входящих в библиотеку выполненных компьютерных моделей.

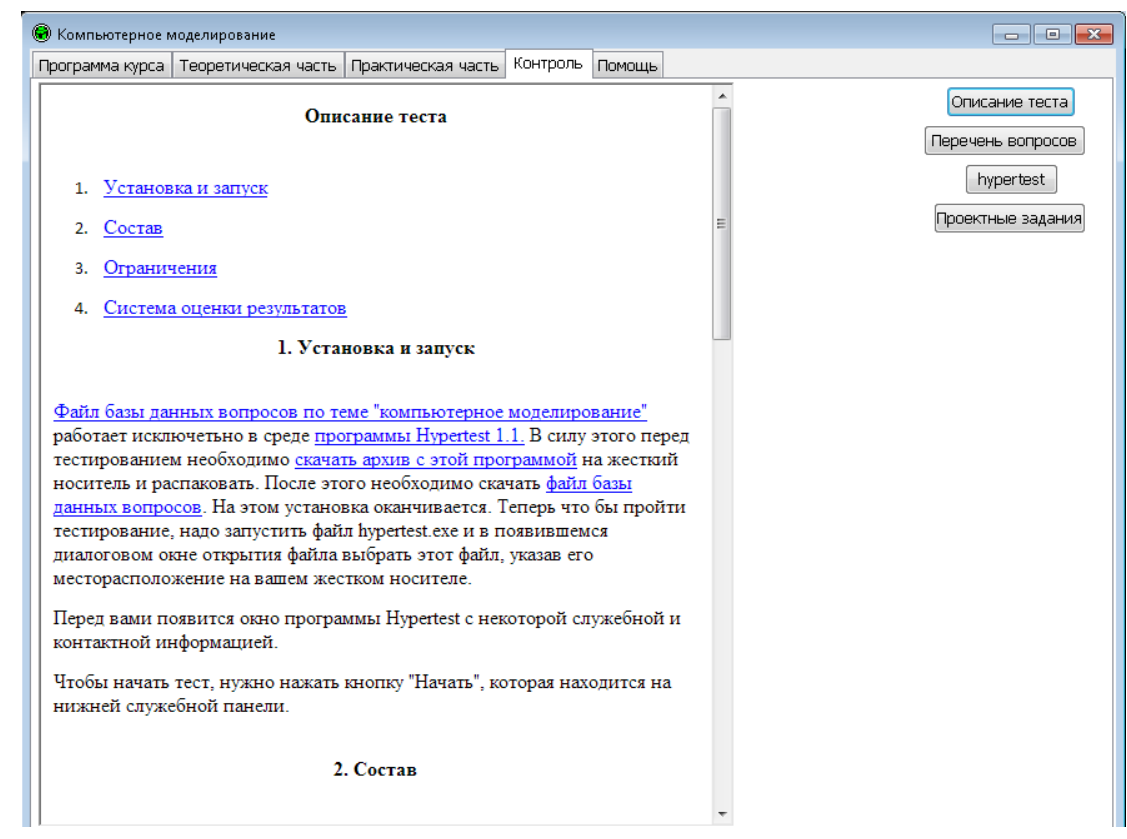

Рисунок 7 - Тестовый вариант контроля. 
Обучение с использованием различных программных комплексов и методик составляет широкий спектор. Данный программный продукт слзданный при помощи среды программирования Delphi, входит в малую часть всех средств обучения. Его использование может помочь обучающемуся в освоении различных сложных разделов компьютерного моделирования с использованием не только теоретической составляющей, но и широкого комплекса практических задач включенного в программу.

Метод проектов строит обучение на активной основе, через целесообразную деятельность обучающегося, сообразуясь с его личным интересом именно в этом знании. Со временем реализация метода проектов претерпела некоторую эволюцию. Родившись из идеи свободного воспитания, она становится в настоящее время интегрированным компонентом вполне разработанной и структурированной системы образования.

Метод проектов и обучение, в сотрудничестве, находят все большее распространение в системах образования разных стран мира. В последнее время этому методу уделяется пристальное внимание и в России. Причинами этого являются необходимость не столько передавать ученикам сумму тех или иных знаний, сколько научить приобретать эти знания самостоятельно, уметь пользоваться приобретенными знаниями для решения новых познавательных и практических задач.

\section{References:}

1. Arkhangel'skiy AY (2003) Programmirovanie v Delphi 7:Binom.- 2003.-pp.1152.

2. Darakhvelidze PG, Markov EP (2003) Programmirovanie v Delphi 7:BKhVPeterburg.-2003.-147.

3. (2015) Delphi Russian Knowledge Base. Available: http://www.drkb.ru/ (Accessed:25.01.15).

4. (2015) Метод проектов. Available: http://www.informatika.kspu.ru/mproj/umk_mo deling/stud_projmeth.php (Accessed:25.01.15).

5. Nikita Kul'tin (2004) Osnovy programmirovaniya v Delphi 8 dlya Microsoft.NET Framework. Samouchitel': BKhV-Peterburg.-2004.
6. Petr Darakhvelidze, Evgeniy Markov (2003) Razrabotka Web-sluzhb sredstvami Delphi: BHV-Sankt-Peterburg.-2003.

7. Boev VD, Sypchenko RP (2010) Komp'yuternoe modelirovanie:InternetUniversitet Informatsionnykh Tekhnologiy, 2010.

8. Bakhvalov LA (2006) Modelirovanie sistem: Uchebnoe posobie dlya vuzov: Izdatel'stvo Moskovskogo gosudarstvennogo gornogo universiteta,2006.

9. Koznov DV (2008) Osnovy vizual'nogo modelirovaniya: Internet-Universitet Informatsionnykh Tekhnologiy; BINOM. Laboratoriya znaniy, 2008.

10. Kel'ton D, Averill M (2004) Imitatsionnoe modelirovanie.-2004. 
